\title{
Schluss: Ein Plädoyer für die Zukunft der Zwischenphase
}

Kehren wir zum Anfang zurück. Die vorangegangenen Ausführungen dienen als Versuch, ein erneutes Nachdenken über die Phase zwischen Goethezeit und Realismus anzustoßen - eine Beschäftigung damit, wie die vielfach konstatierte Vielschichtigkeit und Diversität dieser Phase produktiv in die Literaturgeschichtsschreibung eingebracht werden könnte, um sie nicht einfach kapitulierend als Diffusität abzutun. Die Literaturwissenschaft täte im Gegenteil gut daran, die Differenz im gegebenen Objektbereich als solche anzuerkennen und sie in der Modellbildung des literarhistorischen Segments und der Periodisierung der Literatur des 19. Jahrhunderts zu berücksichtigen.

Nimmt man diese Prämisse ernst und folgt ihr in der Annäherung an den Gegenstand, so ist umso erstaunlicher, dass in Auseinandersetzung mit der Zwischenphase eine Merkmalsmenge erkennbar wird, die das heterogene Feld überlagert: die Relevanz von >Zeit< und insbesondere der reflexive Fokus auf die Denkfigur >Zukunft<. Überspitzt gesagt, macht es keinen Unterschied, ob ein gegebenes Werk eher der stile- und wertekonservativen Richtung oder der Tendenzliteratur zuzurechnen ist, es wird - jedenfalls ist dies bei den hier untersuchten, repräsentativen Texten der Fall - irgendwie >Zeit< in den Fokus gerückt und >Zukunft< ins Auge gefasst; und beides deutlich hervorgekehrt. Die Zwischenphase - das Literatursystem, das von ungefähr 1820 bis 1850 Bestand hat - begreift sich selbst als Phase, als Interim und belegt sich nachgerade selbst mit deutlich markierten temporalsemantischen und temporaldeiktischen Termen, die selbstreflexiv und metatextuell angelegt sind und jene als Teilabschnitt der Literaturgeschichte gleichermaßen von der Goethezeit und vom Realismus abgrenzen. Texte der Zwischenphase enthalten die Bedeutungskomponente >Zeitreflexion< und beziehen sich mit ihr auf den eigenen Status in der Literaturgeschichte. 
Nun geht es dieser Phase allerdings nicht um die Reflexion der Zeit an sich, und wenn doch, dann nur subtil - und das erschwert vorderhand das Unternehmen wie das vorliegende. Eine explizite Zeitrelativierung wie sie Thomas Mann in seinem Zauberberg (1924) vor Augen führt, ein narratoriales Durchbrechen zeitlicher Gesetzmäßigkeiten im Erzählen wie in Ilse Aichingers Die Spiegelgeschichte (1949), ihre literarische > Verwissenschaftlichung « wie in Daniel Kehlmanns Mahlers Zeit (1999) oder die mehrdimensionale Zeitauffächerung in sogenannten >Dimensionslöchern< wie mitunter im Erzählkosmos bei Walter Moers - erklärt im Quasi-Lexikon Zamonien. Entdeckungsreise durch einen phantastischen Kontinent (2012) -, alles dies findet sich hier nicht. Auch Possible Worlds und das literarische Spiel mit alternativen >Zeitwirklichkeiten< wie im Science-FictionRoman Replay (2012) von Benjamin Stein sucht man im gegebenen Zeitraum vergebens. Und doch sind gewisse Anlagen für derartige Phänomene, wie sie in späteren Literatur- und Denksystemen lokalisierbar sind, durchaus vorhanden und benennbar. Wird nicht Zeit schon dann relativiert, wenn Figuren allenthalben über ihre subjektive Zeitgebundenheit sinnieren und zwar in Abgrenzung zur kulturell konzipierten Zeit wie auch zum physikalisch-objektiven Zeitverlauf? Und wird nicht auch ihre Relativität unterstrichen, wenn dieses Gefüge bestehend aus subjektiver Zeit, kultureller Zeit und natürlicher Zeit nicht nur aufgebaut, sondern auch in Frage gestellt wird? Und was den Umgang mit Zukunft betrifft: Wenn doch auch der lebensweltliche Rahmen und das wissenssoziologische Fundament, das etwa Possible Worlds denkbar erscheinen lässt, gänzlich andere sein mögen, als die der Kultur des zweiten Drittels des 19. Jahrhunderts - das, was literarische Texte problematisieren und auszuhandeln versuchen, ist die Unvorhersehbarkeit des Daseins - das, was wir die Imponderabilität von >Zukunft< genannt haben - und zwar ausgelöst durch eine tendenzielle (und unvorhersagbare) Auflösung tradierter Vorstellungen von und Maßgaben für Lebenswege. Davon betroffen sind offensichtlich ganz konkret das Werte- und Normensystem und das Verhaltenssystem, das diese Texte verarbeiten: An welchen Werten und an welchen Normen man sein Handeln und Verhalten auszurichten hat, wird infragegestellt und gerät in einen Zwiespalt zwischen Orientierung an jenem >Alten< und der Setzung neuer, alternativer Optionen. Daran arbeiten sich Figuren, daran arbeiten sich aber auch die Texte selbst ab: Was kann unter bestimmten gegebenen Voraussetzungen realisiert werden und was nicht? Und müssen nicht auch diese Voraussetzungen einer zusätzlichen Prüfung unterzogen werden? Können sie unanfechtbar als gesetzt gelten? Welche unterschiedlichen Möglichkeiten der Realisierung einer Zukunft gibt es dann? Wann ist eigentlich Zukunft wünschenswert oder nicht wünschenswert? Woran macht man das fest? - Dass angesichts dieses Fragenkatalogs und auch angesichts der Tatsache, dass ihn 
die Texte immer wieder aufstellen und geradezu perpetuieren, von einem literarhistorischen Experimentierfeld gesprochen werden kann, wird dann nur allzu verständlich.

Dem Anschein nach jedenfalls machen die Werke - von Ausnahmen wie etwa Mundts Moderne Lebenswirren einmal abgesehen - einen unauffälligen Eindruck. Die Größe >Zeit<, die Reflexion von Zeit und Zukunft sind nicht zwangsläufig als solche ausgestellt (wie bei Mann, Aichinger, Kehlmann, Moers und Stein). Repräsentieren zwar der Zeitroman (Göttsche) und das Phänomen des historischen Erzählens (Sottong) entscheidende Wesenszüge der Literatur der Zeit, so stellen sie doch lediglich die Spitze eines unterhalb der augenscheinlichen Oberfläche sehr viel größeren Eisbergs dar. Zeitreflexion in ihrer spezifischen Verfasstheit reicht weit über diese (wenn auch bedeutsamen) Ausformungen hinaus. Als tragende Säulen sind in dieser Studie die Weiterverarbeitung der goethezeitlichen Initiationsgeschichte, die regressiv-progressive Gestaltung von >Welt $<$, die zeitreflexive Konstitution selbstreflexiven Erzählens und die emphatische Zukunftsausrichtung benannt worden - ohne damit den Anspruch auf Vollständigkeit in der Erfassung des Objektbereichs erheben zu wollen, wenn doch schon den Anspruch auf Repräsentativität. Einen Brennpunkt entsprechender Behandlungen bildet die >heiße< Phase der 1830er-Jahre. Wesentlich sind diese Zeitstrukturen deshalb - und das machte den Dreh- und Angelpunkt aus -, da sie reflexiv ausgerichtet und daher für das Literatursystem signifikant sind. Dabei geht es, so lässt sich konstatieren, um drei kardinale Punkte: (1) Die Abgrenzung von der Goethezeit. Mit enormem Aufwand wird das Vorgängersystem zum Vorgängersystem erklärt. (2) Der literaturästhetische >Aufwand $<$ selbst. $>$ Enorm< gestaltet sich der betriebene Aufwand dahingehend, dass zum einen progressiv-regressive Strukturanordnungen dominieren und eine Vorwärtsgewandtheit stets nur im Verbund mit Rückwärtsgewandtheit gedacht werden kann; >enorm< ist er aber auch insofern, als ein hohes Potenzial an Selbstreflexivität auszumachen ist, anders gelagert zwar als bei der romantischen Selbstreflexivität, aber doch auffallend genug, um auch in dieser Hinsicht von einer reflexiven Standortbestimmung zu sprechen. Und (3) die Expansion des Zukunftshorizontes. Bemerkenswerterweise ist im Rahmen dieser Prozesse die epistemisch-mentale Ausrichtung auf die Zukunft dominant - sie ist ja keineswegs notwendige Folge in diesem Zusammenhang und doch als Teil der Erzähllogik gesetzt und als Erwartungsraum erweitert. Das Literatursystem ist seinem Vergangenheitsbezug zum Trotz als modernes System zu werten, dem ein Fortschrittsglaube eingeschrieben ist. Und genau das macht es so spannend.

Scheinbar zeigt sich die Zwischenphase als > Vexierbild < des modernen Zeitregimes; unverkennbar allerdings ist das offensive Nachdenken über Zeit, der Hang zum Brechen mit der Vergangenheit, zur Archivierung, zur Gegenwartsreflexion 
und Aufspannen von Zukunftshorizonten. Auch hier gilt: Indem die Zwischenphase den Kollektivsingular >Geschichte< zur Geltung bringt und Fortschritt mitdenkt, dabei zwar einen Bruch zur Vergangenheit inszeniert, gleichzeitig jedoch eine betonte Zukunftsorientierung an den Tag legt - und die Gegenwart mit einem Krisenstatus legt -, bestätigt sie das Zeitregime der Moderne, das erst über hundert Jahre später in eine Auflösungsphase gerät. Mittels Zeitreflexion und mittels Zeitreflexion in der vorliegenden Spezifik - unterstreicht die Zwischenphase vielmehr ein kulturelles Zeitregime, das »seine Ausrichtung radikal von der Vergangenheit auf die Zukunft«, seine Wertbindung damit»vom Alten auf das Neue und vom Bekannten auf das Unbekannte, vom Gewesenen auf das noch Werdende und Kommende « (Assmann 2013: 21) umstellt. Inwiefern dies >radikal< geschieht und der >Umstellungsprozess< selbst abläuft - ebendies wird indessen in der Zwischenphase auf den Prüfstand gestellt.

Und genau diese Kontextualisierung der literarischen Zeitreflexion im gegebenen Segment ist auch zu beachten, wenn man einen Ausblick wagt auf Anschlussmöglichkeiten, die sich aus der Untersuchung ergeben.

Ein erster Anschluss könnte in der Betrachtung der Epochenränder liegen. Wie äußern sich zeitreflexive Formen in der Goethezeit und im Realismus? Die Frage ist allein deshalb berechtigt, da Zeit bekanntlich nicht allein in der Zwischenphase eine Rolle spielt, sondern auch im Vorgänger- und Nachfolgesystem. Zum einen sind alle drei Systeme sicher in einem größeren, mentalitätsgeschichtlichen Rahmen zu denken, zum anderen möchten wir aber die Vermutung anstellen, dass sie in ihrer jeweiligen Charakteristik divergieren: ihre strukturelle Anlage mag unter Umständen vergleichbar sein, funktionalisiert sind sie aber anders. Das betrifft Schillers Wilhelm Tell (1804), worin im Stauffacher-Monolog zwar eine erstaunliche Analogie zu einigen hier untersuchten Aspekten vorherrscht, die Ausführungen $\mathrm{zu}>$ Alt $<$ und $>\mathrm{Neu}<$ allerdings klar im Freiheitsdiskurs und der Demokratieerprobung verankert und auf diese hin ausgerichtet sind. Ähnliches kann für Goethes Götz von Berlichingen (1773) angenommen werden: Primär verhandelt wird auch dort die Freiheitsproblematik. Und wenn im Werther (1774) von >Zukunft< die Rede ist, dann im Rahmen einer rein subjektbezogenen Reflexion: Das Subjekt begibt sich mit seiner Liebeskonzeption in einen Bereich, der von der Welt entkoppelt ist, in einen Bereich, in dem >Zukunft< gedacht, nicht aber realisiert werden kann. Dreh- und Angelpunkt ist das Subjekt und sein Scheitern an der Autonomie. Und zum Realismus: In Raabes Chronik der Sperlingsgasse (1856) und Alte Nester (1879/80) ist die Zeitproblematik ebenfalls stark ausgeprägt, ebenso wie in Storms Viola tricolor (1874) und Immensee (1850). Doch sind es nicht hier wie da Verlustgeschichten, die uns die Texte präsentieren, verbunden mit Fragen nach der literarisch adäquaten Erfassung und Wiedergabe von 
$>$ Realität<, die aufgeworfen und verhandelt werden? Kurzum: Sind auf der einen Seite Kontinuitätslinien zu ziehen, die die Systeme durchlaufen, und auf der anderen klare Brüche zwischen den Systemen zu erkennen, die auf eine Goethezeitund eine Realismus-Spezifik schließen ließen?

Ein zweiter Anschluss findet sich gleichfalls mit Blick auf die Epochendiskussion - jedoch mit anderem Fokus. Der Abschnitt, der hier $>Z$ wischenphase $<$ genannt worden ist, ist in der gegenwärtigen Forschung nach wie vor höchst umstritten: Periodisierungen sind widersprüchlich, Fragen des Umgangs mit der Heterogenität des literarischen Feldes sind ungeklärt, der Status der Zwischenphase in der Literaturgeschichte ist insgesamt offen. Dabei ist den Verfechtern der Forschung zu den Teilbereichen, die diese Phase ausmachen (Biedermeier, Vormärz, Nachmärz, Junges Deutschland), in ihrem Urteil beizupflichten, es handele sich um äußerst spannende und untersuchungswerte literarische Erzeugnisse - um Werke, deren Behandlung eben offenlegt, wie Literatur im eigenen Selbstverständnis Literaturgeschichte wahrnimmt. Wir wollen meinen, dass die Reflexion von Zeit hier das zentrale Bindeglied zwischen unterschiedlichen Gruppierungen, Strömungen, Ästhetiken und Diskursivierungsmustern darstellt. Ließen sich also Analogien und Binnendifferenzen herausarbeiten, um das Merkmalsset `Zeitreflexion< zu präzisieren? Angestoßen ist damit ebenfalls der Anschluss einer Korpuserweiterung. Seien auch Aussagen über die Novellistik der Zwischenphase repräsentativ für den gegebenen Zeitraum (da sie, wie festgestellt, in einer immensen Produktionsfülle aufgeht), sie sind doch auch restriktiv. Dass aber Zeit ebenfalls eine nicht unerhebliche Rolle in der Lyrik (zum Beispiel bei Droste-Hülshoff oder Heine), im Drama (unter anderem bei Hebbel) und speziell im Roman (bei Immermann, Gutzkow und Mundt) spielt, klang hier und da zwar an. Allerdings kann auch das Unternehmen einer eingehenderen Untersuchung in diesen Teilfeldern an dieser Stelle gleichfalls nur an die künftige Forschung weitergegeben werden.

Und nicht zuletzt der Anschluss an mentalitätsgeschichtliche Prozesse. Im gegebenen Fall: die genauere Bestimmung des Ortes der literarischen Verhandlung von Zeitfragen im Rahmen des modernen Zeitregimes. Zu berücksichtigen wäre dahingehend eine fundierte Auseinandersetzung mit nichtliterarischer Kommunikation, mit Merkmalsverschiebungen im Zeitdiskurs und denkgeschichtlichen Regulationen im Umgang damit. Die Ansätze insbesondere von Koselleck und Assmann bildeten für unsere literaturwissenschaftliche Studie die Rahmung - nun gilt es, diese Rahmung zu durchleuchten und mit den hier getroffenen Ergebnissen abzugleichen.

Angesichts von alledem - der erzielten Ergebnisse und der genannten Anschlussmöglichkeiten - versteht sich dieses Buch letzten Endes im doppelten Sinne als Plädoyer für die Zukunft der Zwischenphase. 
Open Access Dieses Kapitel wird unter der Creative Commons Namensnennung 4.0 International Lizenz (http://creativecommons.org/licenses/by/4.0/deed.de) veröffentlicht, welche die Nutzung, Vervielfältigung, Bearbeitung, Verbreitung und Wiedergabe in jeglichem Medium und Format erlaubt, sofern Sie den/die ursprünglichen Autor(en) und die Quelle ordnungsgemäß nennen, einen Link zur Creative Commons Lizenz beifügen und angeben, ob Änderungen vorgenommen wurden.

Die in diesem Kapitel enthaltenen Bilder und sonstiges Drittmaterial unterliegen ebenfalls der genannten Creative Commons Lizenz, sofern sich aus der Abbildungslegende nichts anderes ergibt. Sofern das betreffende Material nicht unter der genannten Creative Commons Lizenz steht und die betreffende Handlung nicht nach gesetzlichen Vorschriften erlaubt ist, ist für die oben aufgeführten Weiterverwendungen des Materials die Einwilligung des jeweiligen Rechteinhabers einzuholen. 